\title{
Visualizing the propagation of volume magnetization in bulk ferromagnetic materials by neutron grating interferometry (invited)
}

\author{
C. Grünzweig, ${ }^{1, a)}$ C. David, ${ }^{1}$ O. Bunk, ${ }^{1}$ J. Kohlbrecher, ${ }^{1}$ E. Lehmann, ${ }^{1}$ Y. W. Lai, ${ }^{2}$ \\ R. Schäfer, ${ }^{2}$ S. Roth, ${ }^{2}$ P. Lejcek, ${ }^{3}$ J. Kopecek, ${ }^{3}$ and F. Pfeiffer ${ }^{4}$ \\ ${ }^{1}$ Paul Scherrer Institut, CH-5232 Villigen PSI, Switzerland \\ ${ }^{2}$ IFW Dresden, Institute for Metallic Materials, D-01069 Dresden, Germany \\ ${ }^{3}$ Institute of Physics, AS CR, 18221 Praha 8, Czech Republic \\ ${ }^{4}$ Department of Physics, Technical University of Munich, D-85747 Garching, Germany
}

(Presented 19 January 2010; received 30 October 2009; accepted 19 November 2009; published online 5 May 2010)

\begin{abstract}
In this article we report on a neutron interferometry technique based on diffraction gratings which was used to visualize the geometry-dependent magnetization processes in bulk ferromagnetic materials. The contrast origin is based on the refraction of unpolarized neutrons at magnetic domain walls and the obtained image is termed neutron dark-field image (DFI). The magnetization process is imaged by measuring the spatially resolved domain wall density distribution of the sample. The sample under investigation was a polycrystalline steel plate where the magnetization process was imaged for different sample orientations. The DFI results of the magnetization processes were verified on the one hand by complementary neutron small angle scattering (SANS) experiments and on the other hand by finite element method (FEM) simulations. The obtained SANS and FEM results verify the same magnetization process behavior as observed in the DFI results. () 2010 American Institute of Physics. [doi:10.1063/1.3365373]
\end{abstract}

\section{INTRODUCTION}

Bulk ferromagnetic materials are commonly used as hard or soft magnetic material, e.g., as magnetic clamps, magnet yokes, or components of electric motors. Today, there is a variety of imaging techniques to investigate domain structures at the surface of materials and in thin-films. These techniques, which are either based on light, electrons, or x-rays are limited in penetration depth. ${ }^{1}$ Researchers utilize the advantage of neutrons as they can easily penetrate centimeter thick metallic samples to investigate domain structures in bulk materials. This stimulated the development of neutron imaging techniques ${ }^{2}$ which are based on single-crystal interferometry, ${ }^{3-5}$ crystal analyzer-based topography, ${ }^{6,7}$ or on neutron depolarization analysis. ${ }^{8,9}$

A new neutron imaging approach that delivers insight into bulk magnetic domain structures was recently developed. ${ }^{10-12}$ The approach is based on the so called neutron dark-field image (DFI) and relies on a neutron interferometer setup based on diffraction gratings. ${ }^{13}$ The setup is the same as used for recording neutron phase contrast images. ${ }^{14}$ Using the grating interferometry technique we are able to overcome some of the drawbacks that challenge other neutron imaging methods in terms of exposure times and image quality. The technique was used on the one hand to investigate bulk magnetic domain structures ${ }^{11}$ and on the other hand to study bulk magnetization processes. ${ }^{10}$ The relaxed requirements on spatial and temporal coherence of the grating interferometer setup lead to exposure times that are comparable to other "non-neutron" domain observation techniques. The efficiency of our setup, with total exposure times of typically

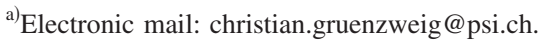

few minutes per DFI, allows us to study the dynamic response of the specimen under the influence of an externally applied magnetic field.

In this article we report about the visualization of the geometry-dependent magnetization processes in a bulk ferromagnetic sample. In the neutron grating interferometry experiments, spatially resolved images of the scattering active areas of the sample, namely, domain wall-rich areas were visualized. The grating interferometer provides therefore local scattering information of the sample. For the visualization of the bulk magnetization process single DFIs were recorded for different magnetic field values. The comparison and verification of the DFI results was made with two independent techniques. On the one hand this was a small angle neutron scattering (SANS) experiment that delivers an integral information whether scattering at domain walls in the sample is present or not and additionally provides information about the domain wall distribution in the sample. The same magnetic field values as for the interferometry measurements have been used for the SANS measurements to provide a direct comparison of both results. On the other hand finite element method (FEM) simulations have been performed. The local magnetic induction field values in the sample for each external field value were obtained. Each FEM image is discussed on the basis of the magnetization curve of the sample in context with phase theory.

\section{SETUP AND CONTRAST ORIGIN}

The interferometer setup consists of three gratings, as schematically shown in Fig. 1(a). ${ }^{15}$ This is the source grating $\mathrm{G}_{0}$ which consists of absorbing lines, a beam splitter or phase grating $\mathrm{G}_{1}$, and an analyzer absorption grating $\mathrm{G}_{2}$. The 


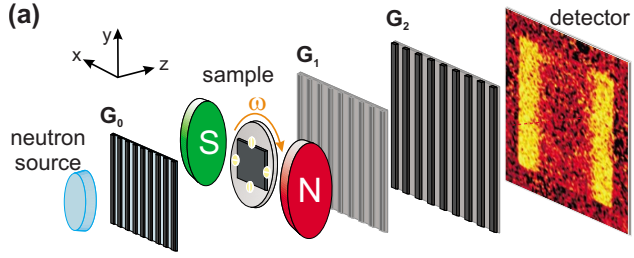

(b)

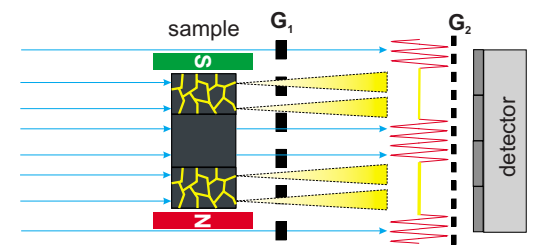

FIG. 1. (Color online) (a) Setup with the source grating $G_{0}$, the phase grating $\mathrm{G}_{1}$, the analyzer absorption grating $\mathrm{G}_{2}$, and an electromagnet creating a horizontal variable magnetic field around the sample. (b) Multiple refraction of neutrons at magnetic domain walls in the sample causes a local degradation of the coherence of the neutron wave field and results in a decrease of the local fringe visibility of the interference pattern.

field of view provided by the gratings can range up to 64 $\times 64 \mathrm{~mm}^{2}$. The moderate temporal coherence requirements of the grating interferometer can be satisfied by using a velocity selector which delivers a bandwidth of $\Delta \lambda / \lambda \simeq 15 \%$ around the design wavelength $\lambda=4.1 \AA$.

To magnetize the samples, we installed a standard dipole electromagnet with cylindrical pole shoes with $40 \mathrm{~mm}$ diameter. The pole gap was $40 \mathrm{~mm}$ producing an almost uniform horizontal magnetic field up to $250 \mathrm{mT}$ with $\Delta H / H<10^{-3}$ in the sample region.

The sample was a polycrystalline steel plate [steel grade: DC 01 (St 12.03)] with an edge length of $15 \mathrm{~mm}$. The plate had a thickness of $750 \mu \mathrm{m}$. The plate was mounted with plastic screws on the front of the sample holder. The sample holder is built in the setup on a three axis positioning system to accurately place the sample centered between the pole shoes. The holder can be rotated around the beam axis by an angle $\omega$, as illustrated in Fig. 1(a) to study the geometrydependent magnetization processes.

The image contrast for magnetic samples, as depicted in
Fig. 1(b), is based on scattering of unpolarized neutrons at magnetic domain walls in the sample. ${ }^{16}$ The neutron beam undergoes multiple refractions at magnetic domain walls in the sample, which results in a local degradation of the coherence of the neutrons exiting the sample. This local degradation decreases the ability of the neutrons to interfere with each other after the phase grating $G_{1}$, and leads to a local decrease of the interference pattern. A detailed description of the DFI contrast can be found in Ref. 17. The exposure time for one DFI was $4 \mathrm{~min}$, and the effective spatial resolution of $100 \mu \mathrm{m}$ was mainly determined by the intrinsic blurring in the scintillation screen. ${ }^{13}$

\section{NEUTRON DARK-FIELD IMAGING RESULTS}

The neutron grating interferometry experiments were carried out at the spallation neutron source SINQ at the Paul Scherrer Institut (PSI) using the beam port of the cold neutron imaging facility ICON. ${ }^{18}$ The results of the geometrydependent magnetization process for different sample angles with respect to external magnetic field are presented in Fig. 2.

The magnetization process of the steel plate for the $\omega=0^{\circ}$ orientation is shown in Fig. 2 (top row). In zero field configuration $H=0 \mathrm{mT}$, the steel plate is clearly and fully visible in the DFI due to the rich multidomain structure in the specimen. When the magnetic field is increased to $H=88 \mathrm{mT}$, contrast starts to regain. At field values of $H \geq 100 \mathrm{mT}$, a vertically elongated region in the middle of the plate becomes visible. In this region the domain wall density is strongly reduced. For increasing field values between $113 \mathrm{mT} \leq H \leq 150 \mathrm{mT}$, this area expands toward the sample edges, gaining rapidly in width. For the maximum external field value of $H=250 \mathrm{mT}$, the larger part of the plate is magnetized beside two small vertical domain wallrich strips at the edges.

For the $\omega=22.5^{\circ}$ orientation, the steel plate is rotated clockwise. When the magnetic field is increased to $H$ $=100 \mathrm{mT}$, the contrast recovers, forming a rectangular, vertically inclined stripe in the middle of the plate. The starting

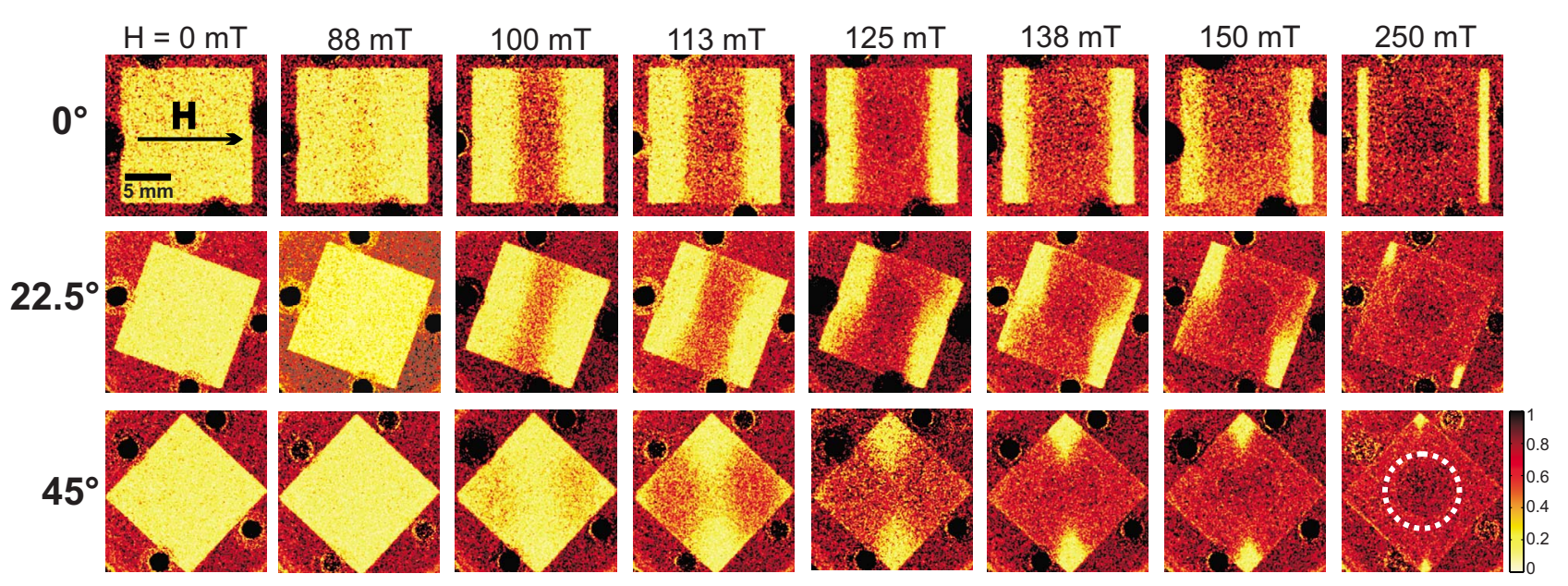

FIG. 2. (Color online) Neutron DFIs of the geometry-dependent magnetization process of the polycrystalline steel plate for different orientations $\omega=0^{\circ}, 22.5^{\circ}$, and $45^{\circ}$. Different magnetization behaviors and starting points of the propagation of the volume magnetization for each orientation are observed. The yellow color in the DFIs indicates domain wall-rich areas. 

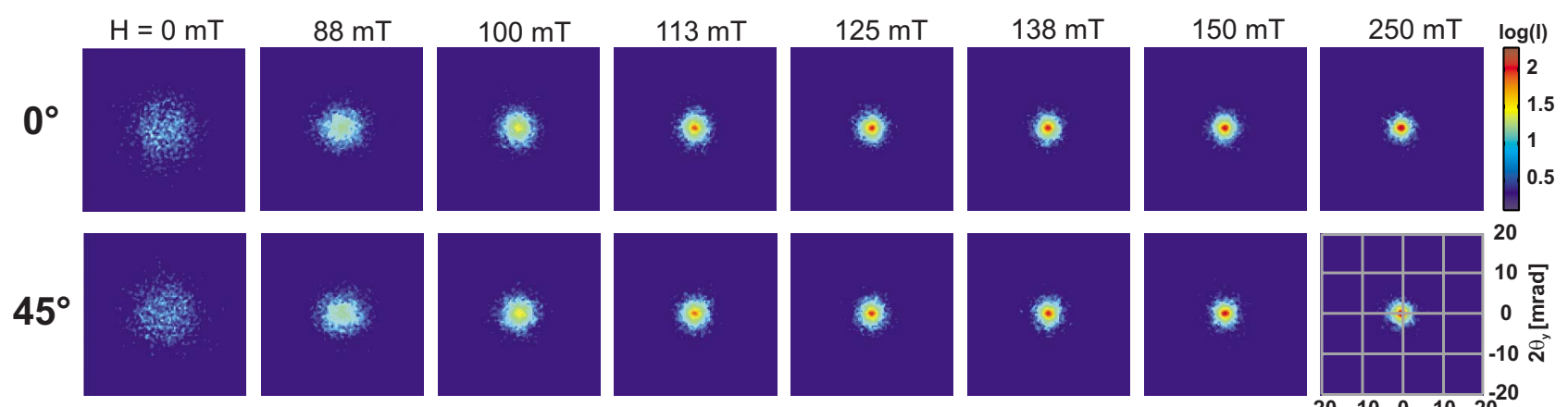

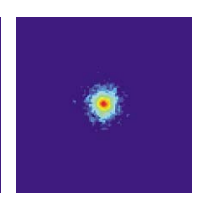

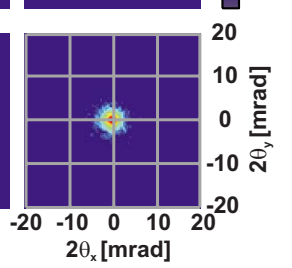

FIG. 3. (Color online) SANS results of the poly-crystalline steel plate for the $\omega=0^{\circ}$ and $45^{\circ}$ orientations and a $H$-field ramp from 0 to 250 mT. In 0 field configuration $H=0 \mathrm{mT}$, for both orientations an isotropic scattering pattern is observed indicating a random domain wall orientation. A collapse of both SANS patterns between $H=88 \mathrm{mT}$ to $H=113 \mathrm{mT}$ is seen. For further increase of the magnetic field the scattering disappears and the original circular beam profile is finally regained at $H=250 \mathrm{mT}$.

position in the middle of the sample is similar to that of the $0^{\circ}$ case. However, the starting point of the contrast development is delayed. At field values between $113 \mathrm{mT} \leq H$ $\leq 150 \mathrm{mT}$, the rectangular area in the middle expands again toward the sample edges and gains rapidly in width. However, at $H=125 \mathrm{mT}$, the rectangular area collapses and for field values between $125 \mathrm{mT} \leq H \leq 150 \mathrm{mT}$, the area expands toward the sample corners which are closer to the pole shoes (top right, bottom left) until these corners are fully magnetized. At maximum field, two small domain wall-rich rectangular areas are remaining on the top left and bottom right corners.

For the $\omega=45^{\circ}$ orientation, the steel plate is oriented with one diagonal along the magnetic field axis. Up to field values of $H \leq 88 \mathrm{mT}$, the steel plate is completely visible in the DFI. For the following magnet field step at $H=100 \mathrm{mT}$ the contrast starts to set in again in the form of two mirrorsymmetrical vertically elongated areas. At $H=113 \mathrm{mT}$, two oval grainy areas are visible, although the horizontal edges close to the pole shoes are still not magnetized. For field values between $113 \mathrm{mT} \leq H \leq 150 \mathrm{mT}$, these two oval areas expand toward the middle part of the plate as well as to the edges close to the pole shoes. The area of the rhombus decreases for increasing external field values. For field $H=250 \mathrm{mT}$, the remaining rhombohedral domain wall-rich areas are found at the top and bottom corners.

\section{SANS RESULTS}

As mentioned the SANS experiments were conducted to verify and to link the DFI results. In contrast to the DFI results of the magnetization process, which deliver information of the scattering signal in real-space, the SANS experiment delivers an averaged signal of the probing area in reciprocal-space.

The SANS experiments were carried out at the SANS I instrument at SINQ at PSI. ${ }^{19}$ A wavelength of $\lambda=18 \AA$ with a bandwidth of $\Delta \lambda / \lambda \simeq 15 \%$ was used. For the measurements the sample was mounted in the same electromagnet as that used for the DFI experiments. The size of the beam at the sample position was $8 \mathrm{~mm}$ in diameter, defined by a cadmium aperture, limiting the measurements to the central part of the steel plate.
The SANS signal for the disk was measured with the same ramping of the external magnetic field as used for the interferometry experiments $(0 \mathrm{mT} \leq H \leq 250 \mathrm{mT})$. The SANS results for the $\omega=0^{\circ}$ and $\omega=45^{\circ}$ orientations and for increasing external magnetic field from $H=0 \mathrm{mT}$ to $H=250 \mathrm{mT}$ are shown in Fig. 3. Due to the scattering of neutrons at the domain walls in the steel plate, for both orientations, a wide symmetric circular diffraction pattern is observed when no magnetic field is applied. The sample is in a complex multidomain configuration state and covers all wall orientation directions in space due to the non oriented nature of the steel plate.

For the $\omega=0^{\circ}$ orientation a rapid transition of the diffraction pattern is observed at field values of $88 \mathrm{mT} \leq H$ $\leq 113 \mathrm{mT}$, where the wide spread diffraction pattern reduces to well defined circular diffraction pattern. For $\omega$ $=45^{\circ}$ a similar transition as for the $0^{\circ}$ case is observed. A further increase of the external field for both orientations delivers the original beam profiles. The measured SANS detector pattern for the $45^{\circ}$ case basically resembles that of the $0^{\circ}$ orientation, so that the orientation of the sample could not been distinguished within the SANS measurements. This can be explained by the randomly oriented domain walls in the sample as expected for a polycrystalline material. As mentioned, the probing size of the neutron beam for the SANS measurements was $8 \mathrm{~mm}$ in diameter (compare white circle in the DFI for $\omega=45^{\circ}$ and $250 \mathrm{mT}$ in Fig. 2). Consequently, if domain wall-rich regions are located outside of the probing SANS area (white circle), these regions are not contributing to the SANS signal. For the $0^{\circ}$ case and at $H=125 \mathrm{mT}$, the domain wall-free vertical stripe in the DFI in Fig. 2 already has a width larger than $8 \mathrm{~mm}$, e.g., the outer parts (yellow stripes) no longer contribute to the SANS signal and the scattering pattern stay constant for increasing field values. Regarding the $45^{\circ}$ case, the constant SANS pattern is observed at $H=138 \mathrm{mT}$ as the probing area seen in the corresponding DFI is free of domain walls.

The strong link between the SANS and the DFI results can also be qualitatively observed by comparing Figs. 2 and 3. In both, the SANS and the DFI measurements, a rapid change on going from $H=88 \mathrm{mT}$ to $H=125 \mathrm{mT}$, is observed. Therefore, the SANS signal gives information 


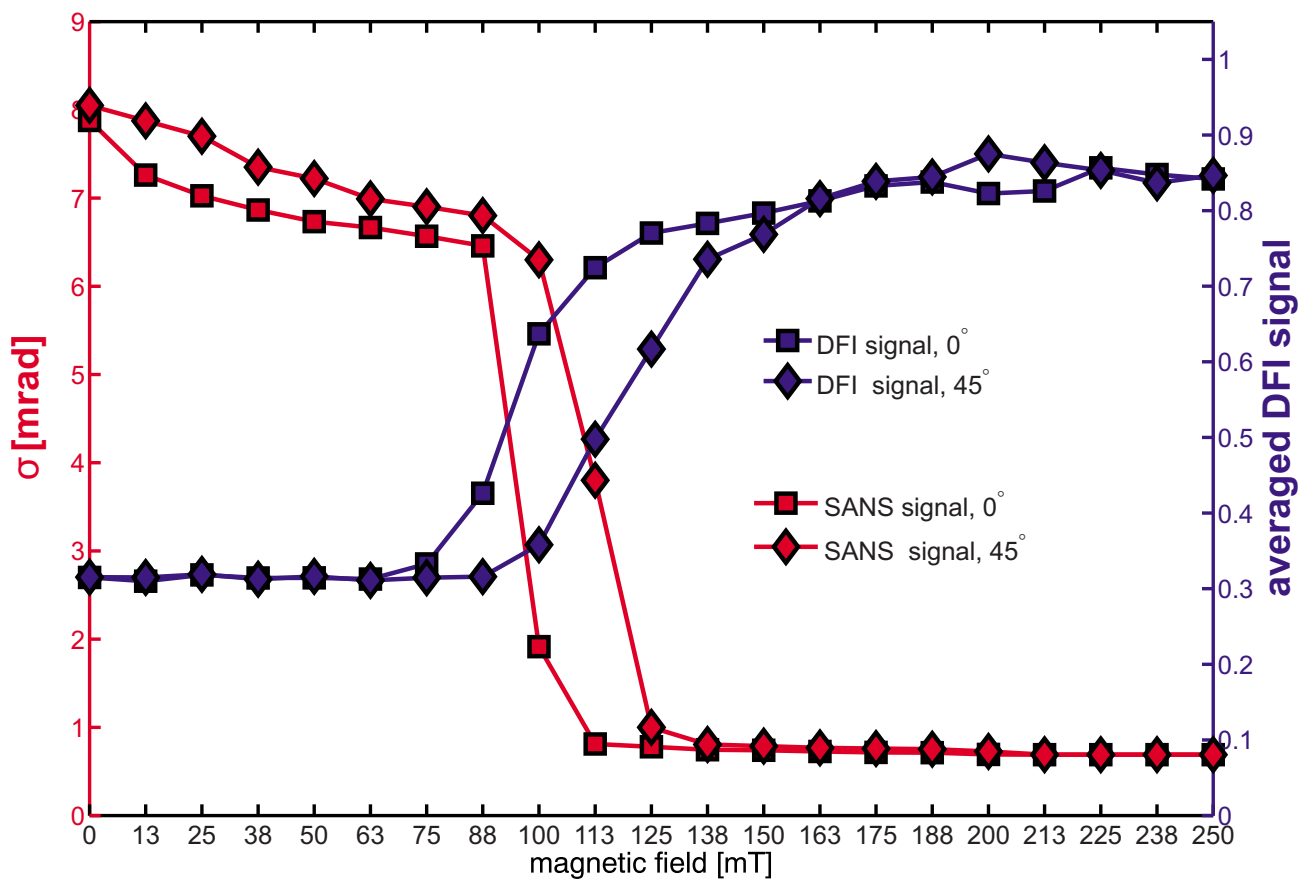

FIG. 4. (Color online) Comparison of the SANS measurement (red line) for the steel plate at $0^{\circ}$ (squared markers) and $45^{\circ}$ (diamond markers) and integrated DFI signal (blue lines) as a function of the external magnetic field from $0 \mathrm{mT} \leq H \leq 250 \mathrm{mT}$. The averaged DFI values are obtained by averaging over image values in a central region of $7.5 \times 7.5 \mathrm{~mm}^{2}$. The SANS values represent the width $(\sigma$-value) of corresponding Gaussian fits.

whether there is a change in the total scattering power in the probing area of the sample and the DFI gives information where the changes locally originate from.

A quantitative analysis is made in Fig. 4 which shows a direct comparison of the measured scattering in the SANS experiments and the averaged DFI signal as a function of the externally applied magnetic field for the $0^{\circ}$ and $45^{\circ}$ orientations. The SANS value represents the width of the Gaussian fit ( $\sigma$-value), obtained by a radial averaging of the corresponding SANS pattern. The displayed integrated DFI signal is obtained by averaging each DFI image over a central 7.5 $\times 7.5 \mathrm{~mm}^{2}$ region of the steel plate. For both, the DFI signal and the SANS signal, the results are plotted when the magnetic field was ramped from $H=0 \mathrm{mT}$ to $H=250 \mathrm{mT}$.

In the multi domain configuration, for $0 \mathrm{mT} \leq H$ $\leq 75(88) \mathrm{mT}$ (bracket values are for the $45^{\circ}$ case), a strong and slowly decreasing scattering signal is observed. This is reflected in the DFI values, which decrease to a stable minimum. Between 75(88) $\mathrm{mT} \leq H \leq 125(138) \mathrm{mT}$, rapid changes in both the DFI and the SANS signals can be observed. These changes are due to the transition of the sample from a largely demagnetized configuration to a magnetized one. The separation of both curves indicates that the magnetization processes are different for each orientation. For the $0^{\circ}$ case, the curves of both the SANS and DFI show an earlier transition than that of the $45^{\circ}$ case. In other words, the steel plate in $45^{\circ}$ orientation needs a higher external field to be magnetized. When the sample is magnetized in the probing area, at $113(125) \mathrm{mT} \leq H \leq 250 \mathrm{mT}$, no scattering is observed in the SANS patterns and the $\sigma$-value for the width of the SANS pattern reduces to the instrument resolution. This is also reflected in the DFI signal, which is close to unity since the probing area is free of domain walls for both cases.

\section{FEM RESULTS}

For the comparison and verification of our measured DFI data presented in Fig. 2, the DFI results are compared with FEM simulation results. The FEM analysis is a numerical technique for obtaining approximate solutions of boundaryvalue problems such as partial differential equations as well as integral equations of mathematical physics. ${ }^{20}$ In our case, these are the solutions of the Maxwell equations for the magnetostatic case. We used the commercially available AMPERES 6.0 software which is a three-dimensional (3D) magnetic field solver for such applications. Using this FEM software, we are able to simulate, in three dimensions, the internal magnetic induction $B$ for a given sample and external magnetic field geometry. The input parameters for the simulations are the permeability and the saturation magnetization.

To compare the FEM simulations with the DFI results, the sample is considered as a "large sample" where the contribution of individual domain walls will be completely negligible. ${ }^{1}$ For large samples, the detailed geometrical arrangement of domain patterns is ignored, but the spatial periods of the implied domains must be smaller compared to the sample size in all dimensions, which holds in our case. The only theoretical method to model magnetization processes of such "large samples," is an approach based on "phase theory." similar sense as phases in metallography or in thermodynamics. All domains magnetized in the same direction are gathered into a phase, characterized only by its volume and its magnetization direction. This approximation is valid if the sample is sufficiently large in at least one dimension.

For the FEM simulations, the simulated $B$-field distribution of the magnetization process is discussed in context with 


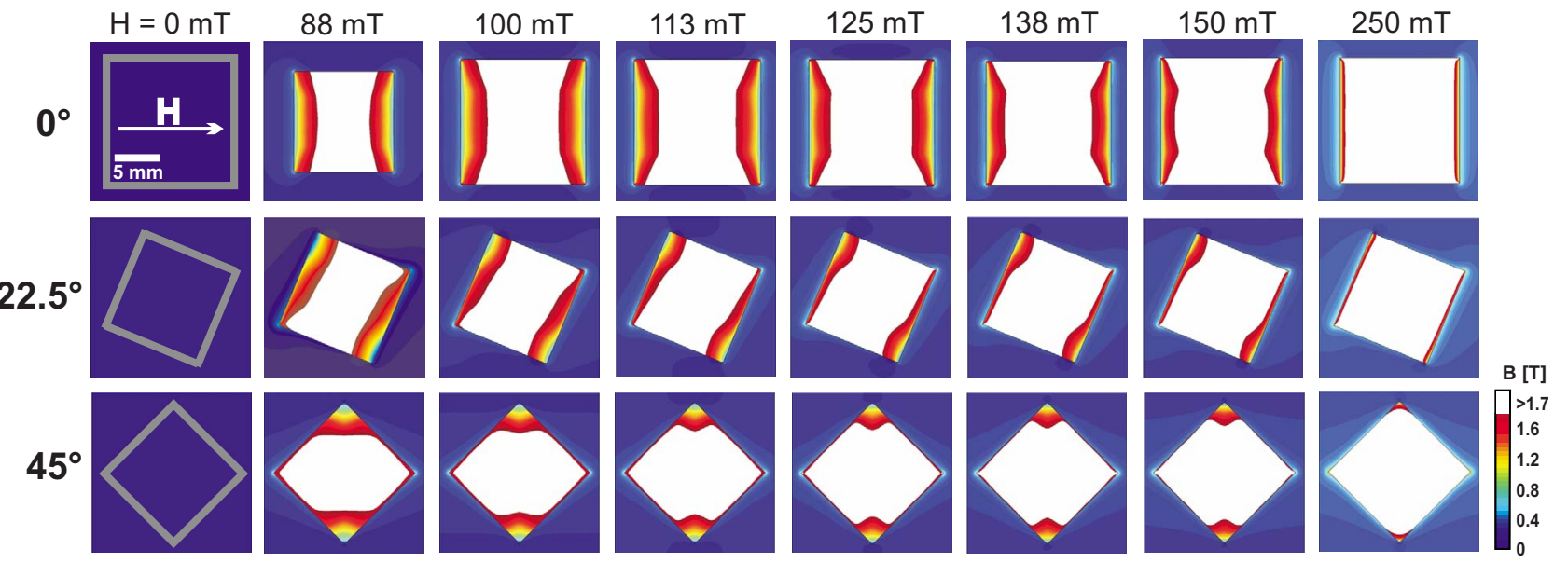

FIG. 5. (Color online) FEM simulation results for the $\omega=0^{\circ}, 22.5^{\circ}$, and $45^{\circ}$ orientations of the steel plate and external field values of $0 \mathrm{mT} \leq H$ $\leq 250 \mathrm{mT}$. White areas in the simulation are associated with Mode II $(B>1.7 \mathrm{~T})$, where the external field penetrates the sample and no more domain walls are present and no scattering of neutrons is present.

phase theory on the basis of the magnetization curve of the steel. The measurement of the $M-H$ curve can be found in Ref. 17. From the measurement the intrinsic permeability of the material is deduced to $\mu \approx 156$ and the saturation magnetization to $M_{S}=2.1 \mathrm{~T}$.

In the magnetization curve, two different modes are distinguished. In Mode I, changes in the magnetization occur by a redistribution of domains. This domain motion is associated with a rapid increase in the magnetization curve. The domain wall displacement characterizing Mode I only takes place at small and moderate external field values. Mode II is associated with larger field values where rotation processes of the magnetization directions out of the easy magnetization axes of the phases are dominate. Concerning the magnetization curve, only a slow increase of $B$ occurs. The characteristic point in the magnetization curve is the transition point, called the "knee," which separates the two modes. Above the knee, the external field penetrates the sample and there are no longer domain walls left and only magnetization rotation takes place. Below the knee, the external field is expelled and only domain wall motion occurs. In single crystalline specimens these two processes occur one after the other but in polycrystalline materials some overlap occurs. The knee for the steel plate material is given at $M_{K}=0.83 M_{S}$ $\approx 1.7 \mathrm{~T}$ for the polycrystalline steel plate material. The value 0.83 is a statistical factor giving the ratio between saturation magnetization and remanent magnetization in polycrystalline materials with cubic anisotropy. ${ }^{1}$

For the simulation, we considered a homogenous cylindrical magnetic field, as created by the pole shoes with a diameter of $40 \mathrm{~mm}$ and a field length of $40 \mathrm{~mm}$ (gap length). The simulations show the local internal magnetic induction values as a function of the externally applied magnetic field values for $0 \mathrm{mT} \leq H \leq 250 \mathrm{mT}$. The results of the FEM simulations for the $0^{\circ}, 22.5^{\circ}$, and $45^{\circ}$ orientations of the steel plate are shown in Fig. 5. The simulated $B$ field images are scaled to $M_{K} \approx 1.7 \mathrm{~T}$. Every $B$ field value larger than $M_{K}$ appears as white in the simulation results. The white color is associated with areas where Mode II is dominating. In these areas no domain walls are left and correspondingly no scattering can occur.
It is seen in the FEM simulation that for the different sample orientations $\left(0^{\circ}, 22.5^{\circ}\right.$, and $\left.45^{\circ}\right)$ the simulated $B$ field distributions appear different. Comparing the FEM simulations with the DFI measurements, the FEM simulations are qualitatively reflect the DFI results, where the trend of the magnetization behavior is reproduced in each of the simulations. For all three orientations, it is imperative that for larger field values the simulation results agree better than for the lower ones. Considering the $0^{\circ}$ case in Fig. 5, the tendency that the sample starts to magnetize from the center is clearly followed, as well as the increase of the magnetized area in horizontal direction. The curvatures of the white areas, as shown in the simulations, are not obtained in the DFIs. In the $22.5^{\circ}$ case, the magnetization behavior as simulated in the FEM images can also be seen in the DFIs. Both the inclined domain wall-free area and the growing of these areas are seen. In the $45^{\circ}$ case, the deviations between the FEM results and the DFI results are largest. At larger $H$ fields, the diamond shaped areas at the top and bottom edges collapse, which is not observed in the corresponding DFI images. The starting points of magnetization processes for the three different orientations are not reproduced in the FEM simulations.

There is an interesting similarity between our bulk DFI observations and magnetization studies in magnetic thinfilms. DeSimone et al. ${ }^{22}$ studied square-shaped Permalloy thin-film elements in diagonal magnetic fields, both experimentally by Kerr observation and by micromagnetic simulations. They found that beyond a certain field value the magnetic field starts to penetrate the element. The local distribution of the inhomogeneously penetrating field, as well as its spatial expansion with increasing field, are analog to the red areas appearing in the DFI measurements on our steel plate at $45^{\circ}$-orientation. In the thin-film elements it was further found that domains and domain hysteresis does only occur in areas where the field did not penetrate, i.e., where the internal field is still 0 . This comparison supports our grating interferometry measurements: in the DFI we visualize the penetration of magnetic field and in the penetrated areas are free of magnetic domain walls. 


\section{CONCLUSION AND OUTLOOK}

In this article it has been shown how a grating-based neutron interferometer can provide spatially resolved images of the internal magnetic domain wall distribution. This was used to image the geometry-dependent magnetization processes of a bulk polycrystalline plate. Complementary SANS experiments validate the interpretation that the loss of spatial coherence revealed in the DFI is caused by the refraction of unpolarized neutrons at magnetic domain walls in the specimen. SANS measurements of the magnetization processes underline the strong link between SANS and DFI results. For the SANS measurements the same magnetization behavior was observed as for the DFI results. The DFI magnetization data have been additionally supported by FEM simulations which show a qualitative accordance. Since the grating interferometer setup is highly efficient because it is compatible with a broad angular and energy spectrum of the incident neutron beams, it opens the way for systematic tomographic investigations of the magnetic properties of bulk materials. We envisage that the method can be extended to 3D using computed tomography and yield 3D images of the magnetic domain wall distributions of bulk ferromagnetic materials of any geometry.

\footnotetext{
${ }^{1}$ A. Hubert and R. Schäfer, Magnetic domains (Springer, Berlin, 1998).

${ }^{2}$ O. Schärpf and H. Strothmann, Phys. Scr., T T24, 58 (1988).

${ }^{3}$ M. Schlenker, W. Bauspiess, W. Gräff, U. Bonse, and H. Rauch, J. Magn.
}

Magn. Mater. 15-18, 1507 (1980).

${ }^{4}$ S. Nakatani, H. Tomimitsu, T. Takahashi, T. Takahashi, and S. Kikuta, Jpn. J. Appl. Phys., Part 2 31, L1137 (1992).

${ }^{5} \mathrm{H}$. Rauch and S. A. Werner, Neutron Interferometry (Oxford University Press, Oxford, 2000).

${ }^{6}$ M. Schlenker and J. Baruchel, J. Appl. Phys. 49, 1996 (1978).

${ }^{7}$ J. Baruchel, Physica B 192, 79 (1993).

${ }^{8}$ M. Schlenker and C. G. Shull, J. Appl. Phys. 44, 4181 (1973).

${ }^{9}$ M. T. Rekveldt, Z. Phys. 259, 391 (1973).

${ }^{10}$ C. Grünzweig, C. David, O. Bunk, M. Dierolf, G. Frei, G. Kühne, J. Kohlbrecher, R. Schäfer, P. Lejcek, H. Rønnow, and F. Pfeiffer, Phys. Rev. Lett. 101, 025504 (2008).

${ }^{11}$ C. Grünzweig, C. David, O. Bunk, M. Dierolf, G. Frei, G. Kühne, R. Schäfer, S. Pofahl, H. Rønnow, J. F. van der Veen, and F. Pfeiffer, Appl. Phys. Lett. 93, 112504 (2008).

${ }^{12}$ M. Strobl, C. Grünzweig, A. Hilger, I. Manke, N. Kardjilov, C. David, and F. Pfeiffer, Phys. Rev. Lett. 101, 123902 (2008).

${ }^{13}$ C. Grünzweig, G. Frei, E. Lehmann, G. Kühne, and C. David, Rev. Sci. Instrum. 78, 053708 (2007).

${ }^{14}$ F. Pfeiffer, C. Grünzweig, O. Bunk, G. Frei, E. Lehmann, and C. David, Phys. Rev. Lett. 96, 215505 (2006).

${ }^{15}$ C. Grünzweig, F. Pfeiffer, O. Bunk, T. Donath, G. Kühne, G. Frei, M. Dierolf, and C. David, Rev. Sci. Instrum. 79, 053703 (2008).

${ }^{16}$ O. Schärpf, J. Appl. Crystallogr. 11, 626 (1978).

${ }^{17}$ C. Grünzweig, Dissertation, ETH Zurich, Switzerland, 2009, 18612.

${ }^{18}$ G. Kühne, G. Frei, E. Lehmann, and P. Vontobel, Nucl. Instrum. Methods Phys. Res. A 542, 264 (2005).

${ }^{19}$ J. Kohlbrecher and W. Wagner, J. Appl. Crystallogr. 33, 804 (2000).

${ }^{20}$ O. C. Zienkiewicz and R. L. Taylor, The Finite Element Method. Its Basis and Fundamentals (Butterworth-Heinemann, Burlington, 2005).

${ }^{21}$ E. Kneller, Ferromagnetismus (Springer, Berlin, 1962).

${ }^{22}$ A. DeSimone, R. V. Kohn, S. Müller, F. Otto, and R. Schäfer, Proc. R. Soc. London, Ser. A 457, 2983 (2001). 